\title{
Reducing expression of synapse-restricting protein Ephexin5 ameliorates Alzheimer's-like impairment in mice
}

\author{
Gabrielle L. Sell, ${ }^{1,2}$ Thomas B. Schaffer, ${ }^{1}$ and Seth S. Margolis ${ }^{1,2}$ \\ 'Department of Biological Chemistry and ${ }^{2}$ Solomon H. Snyder Department of Neuroscience, The Johns Hopkins University School of Medicine, Baltimore, Maryland, USA.
}

\begin{abstract}
Accumulation of amyloid- $\beta(A \beta)$ protein may cause synapse degeneration and cognitive impairment in Alzheimer's disease (AD) by reactivating expression of the developmental synapse repressor protein Ephexin 5 (also known as ARHGEF15). Here, we have reported that $A \beta$ is sufficient to acutely promote the production of Ephexin 5 in mature hippocampal neurons and in mice expressing human amyloid precursor protein (hAPP mice), a model for familial AD that produces high brain levels of $A \beta$. Ephexin 5 expression was highly elevated in the hippocampi of human $A D$ patients, indicating its potential relevance to AD. We also observed elevated Ephexin 5 expression in the hippocampi of hAPP mice. Removal of Ephexin5 expression eliminated hippocampal dendritic spine loss and rescued AD-associated behavioral deficits in the hAPP mice. Furthermore, selective reduction of Ephexin 5 expression using shRNA in the dentate gyrus of presymptomatic adolescent hAPP mice was sufficient to protect these mice from developing cognitive impairment. Thus, pathological elevation of Ephexin 5 expression critically drives $\mathrm{A} \beta$-induced memory impairment, and strategies aimed at reducing Ephexin 5 levels may represent an effective approach to treating AD.
\end{abstract}

\section{Introduction}

Alzheimer's disease (AD) is an age-related neurodegenerative illness characterized by progressive memory loss, impairment of cognitive function, and the formation of amyloid plaques and neurofibrillary tangles $(1,2)$. Accumulation of the amyloid- $\beta(A \beta)$ protein is considered a precipitating event in the pathogenesis of $\mathrm{AD}$ (3). While increased $A \beta$ production is thought to disrupt neuronal function and ultimately lead to cell death (4), neuropathological studies have established excitatory synaptic loss as the best correlate of the pattern and severity of the cognitive deficits observed in $\operatorname{AD}(5,6)$. While these findings demonstrate a crucial role for $\mathrm{A} \beta$-induced aberrations in synapse density $(7,8)$, the downstream events that lead to synaptic dysfunction and $\mathrm{AD}$ pathophysiology remain poorly understood.

Work from our laboratory focuses on identifying and studying factors that operate early in brain development to restrict the number of excitatory synapses that form in order to prevent ectopic synapse formation. Remarkably, studies in the hippocampus of adult patients with early stages of $\mathrm{AD}$ have identified elevated expression of one of these critical factors, Ephexin5 (also known as ARHGEF15), raising the possibility that synaptic loss in $\mathrm{AD}$ may stem from a reactivation of Ephexin5-mediated synapse restriction $(9,10)$.

Ephexin5 is a GEF that activates the small G protein RhoA, a regulator of the actin cytoskeleton. Genetic loss- and gain-of-function studies indicate that Ephexin5 acts to restrict spine growth and synapse development in the developing brain. Upon induction

Conflict of Interest: The authors have declared that no conflict of interest exists. Submitted: November 10, 2015; Accepted: January 24, 2017. Reference information: / Clin Invest. 2017;127(5):1646-1650 https://doi.org/10.1172/JCI85504. of synapse formation, Ephexin5 is phosphorylated by the receptor tyrosine kinase EPH receptor B2 (EPHB2) and targeted for ubiquitin-mediated proteasome-dependent degradation by the E3 ubiquitin ligase UBE3A. Moreover, Ephb2-KO mice show elevated Ephexin5 expression (10).

A $\beta$-triggered disruption of EPHB2 signaling is now recognized as a central feature of $\mathrm{AD}$ pathology $(11,12)$. We hypothesized that loss of EPHB2 signaling in AD could lead to increased Ephexin5, thus contributing to various aspects of $A \beta$-induced deficits. Here, we confirm our hypotheses and show that elevation of Ephexin5 expression does occur in samples from patients with AD and in mouse models of $\mathrm{AD}$. Moreover, we show that a reduction of Ephexin5 expression in the hippocampus of AD-transgenic mice prevents memory impairment. These observations support a key role for Ephexin 5 in the $A \beta$-induced synapse and cognitive pathogenesis that is relevant to $\mathrm{AD}$.

\section{Results and Discussion}

To determine whether $A \beta$ can lead to elevation of Ephexin5 expression, we treated mouse hippocampal neurons with soluble $\mathrm{A} \beta_{1-42}$ during development, when EPHB2 is known to be active and Ephexin 5 is degraded $(10,13)$. We observed an elevation of Ephexin5 levels in $A \beta_{1-42}$-treated neurons as measured by immunocytochemistry (Figure 1A). In addition, we observed that $A \beta$ induces a decrease in EPHB2, a known suppressor of Ephexin5 expression at the surface of neurons (Supplemental Figure 1A; supplemental material available online with this article; https://doi.org/10.1172/ JCI85504DS1). To determine whether $A \beta_{1-42}$ can acutely increase Ephexin5 expression after development and in vivo, we stereotactically injected $A \beta_{1-42}$ into the dentate gyrus (DG) of WT mice and prepared lysates from these hippocampi. Western blot anal- 
A Control
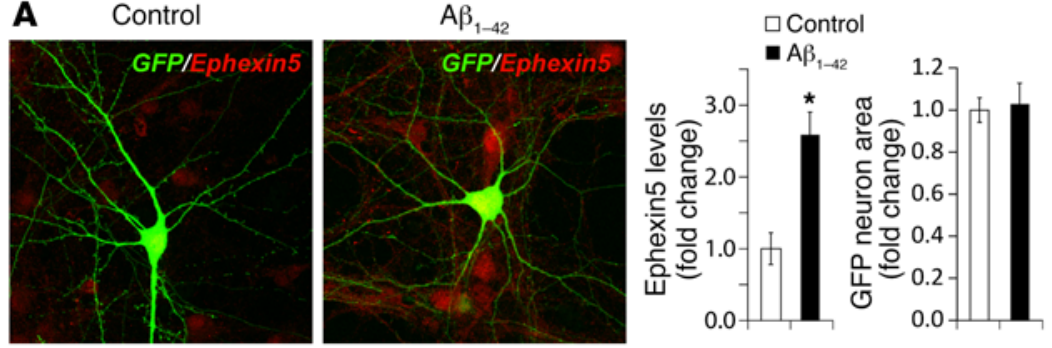

B

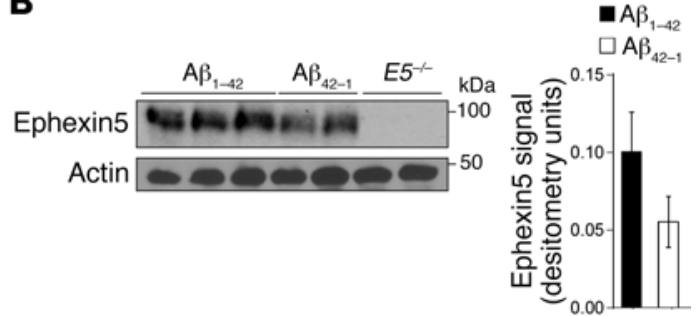

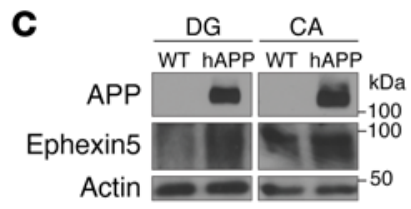
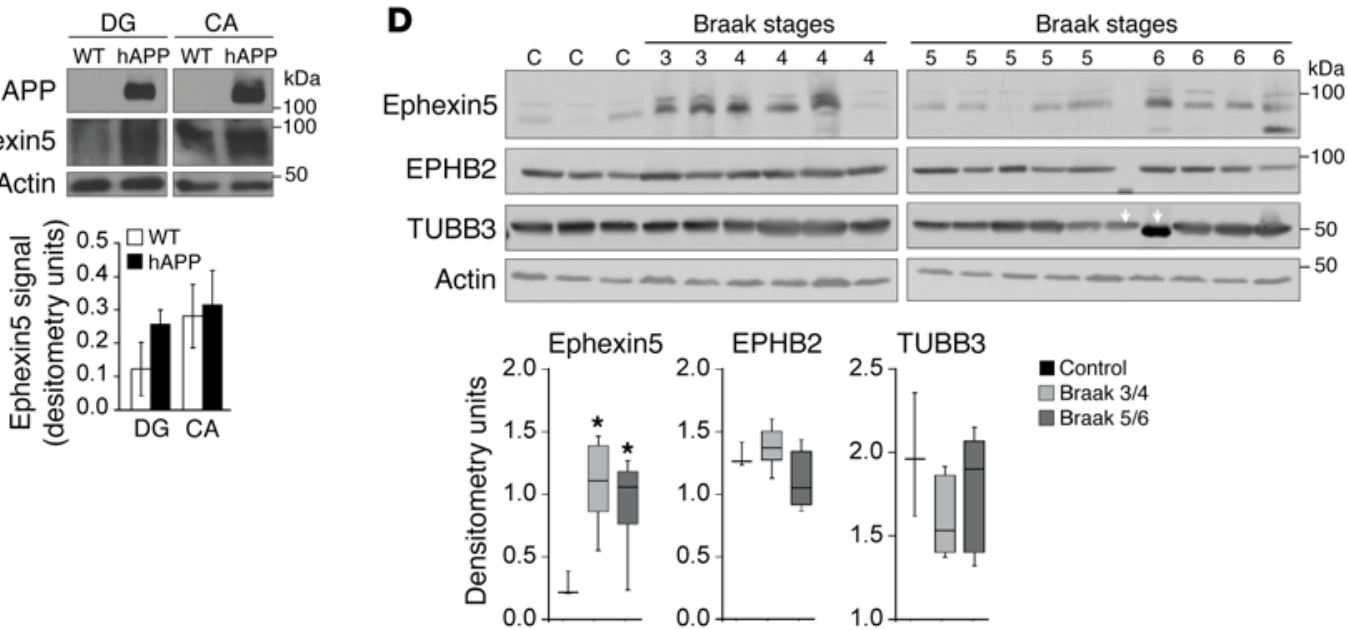

Figure 1. Ephexin5 expression is altered in response to A $\boldsymbol{\beta}$ and in AD. (A) Immunocytochemistry of Ephexin5 from cultured hippocampal neurons treated with control or $A \beta$ peptide. Representative images are shown. Original magnification: $\times 40$. Quantification indicates the intensity of Ephexin 5 signal within GFP-filled neurons ( $n=30$ neurons from 3 independent experiments). ${ }^{*} P<0.05$, by 2-tailed $t$ test. Ephexin5 intensity is shown as Fold change in fluorescence intensity of Ephexin5 signal normalized to control. GFP neuron area is shown as fold change in fluorescence area normalized to control. (B) Western blot of whole hippocampal lysate after injection in vivo of $A \beta_{1-42}$ or $A \beta_{42-1}$, with actin used as a loading control. (C) Representative Western blot of Ephexin5 levels in lysed, microdissected WT $(n=7)$ and hAPP $(n=7)$ CA and DG regions of hippocampus. Ephexin5 levels were normalized to actin. (B and C) Quantification shows arbitrary densitometry units of Ephexin5 signal normalized to Actin loading. (D) Western blot of Ephexin5, EPHB2, TUBB3, and actin in human hippocampal samples ( $C=3$ independent controls; the other samples are marked by Braak stage). Quantifications were normalized to actin. Ephexin5, EPHB2 or TUBB3 signal each normalized to Actin loading (Arbitrary densitometry units). Data in $\mathbf{D}$ are reported in the box and whisker plot. ${ }^{*} P<0.05$, by 1 -way ANOVA with Dunnet's correction.

ysis using antibodies that detect Ephexin5 showed an elevation of Ephexin5 expression in $\mathrm{A} \beta_{1-42}$-injected mice (Figure 1B).

Transgenic mice overexpressing human amyloid precursor protein (hAPP) with familial AD mutations (hAPP mice) have high levels of $A \beta$ production in the brain and reduced surface EPHB2 levels (Supplemental Figure 1B) (11). Consistent with our findings that exogenous addition of $A \beta$ leads to elevated Ephexin5, lysates from hippocampi of WT and hAPP mice showed elevated Ephexin5 expression in hippocampi from hAPP mice (Supplemental Figure 1C). We decided to microdissect hippocampi from hAPP mice, because previous studies had attributed region-specific changes to the effects of $A \beta$ on hippocampal function (14). We found that elevated Ephexin5 was most prominent in the DG, while levels in the cornu ammonis (CA) were not similarly affected by hAPP production between genotypes (Figure 1C).

Taken together, these data indicate a potential link between Ephexin5 expression and AD. Therefore, using antibodies raised against Ephexin5 (Supplemental Figure 1D), we measured Ephexin 5 expression in hippocampi of AD patients at various Braak stages. We observed a 2- to 3-fold increase in the level of Ephexin5 expression at all Braak stages as compared with levels in controls (Figure 1D and Table 1).
Ephexin5 overexpression leads to suppression of dendritic spine formation early during neuronal development (10). Throughout life, hippocampal dendritic spines undergo continual dynamic changes that closely correlate with learning and memory and are profoundly affected in AD $(15,16)$. Transgenic hAPP animals exhibit pathological disruption of dendritic spine density and cognitive impairment $(11,16,17)$. To test the possibility that elevated Ephexin5 in hAPP mice contributes to reduced spine density and cognitive impairment, we crossed hAPP mice with Ephexin5-KO mice $\left(E 5^{-/}\right.$mice). Moreover, $E 5$ deletion did not alter total APP levels in CA, DG, or whole hippocampi of hAPP mice, nor did it alter hippocampal soluble $\mathrm{A} \beta$ levels or plaque formation (Supplemental Figure 2, A-C).

Because we previously observed an increase in Ephexin5 expression in the DG of hAPP mice, we first analyzed spine density and morphology in vivo from the molecular layer of DG from WT, hAPP, hAPP $/ E 5^{--}$, and $E 5^{--}$mice crossed with Thy1-EGFP mice, which express EGFP specifically within a subset of neuronal cells (Figure 2A). Consistent with previous reports showing decreased synapses in the DG of hAPP mice $(18,19)$, we observed a decrease in postsynaptic spine density in hAPP mice in the DG, while hAPP/ $\mathrm{E}^{-/}$mice showed rescued spine density to a degree similar to that 
Table 1. Sex, age, brain region, and postmortem interval for all human samples, organized by Braak stage

\begin{tabular}{|c|c|c|c|c|}
\hline Braak stage & Age (yr) & Sex & PMI & Region \\
\hline Con & 85 & $M$ & 20.83 & Hipp \\
\hline Con & 96 & M & 24.28 & Hipp \\
\hline Con & 106 & M & 21 & Hipp \\
\hline 3 & 82 & $\mathrm{~F}$ & 17.37 & Hipp \\
\hline 3 & 86 & $\mathrm{~F}$ & 10.6 & Hipp \\
\hline 4 & 93 & $M$ & 16.05 & Hipp \\
\hline 4 & 80 & $M$ & 12.37 & Hipp \\
\hline 4 & 98 & $\mathrm{~F}$ & 21.17 & Hipp \\
\hline 4 & 87 & $\mathrm{~F}$ & 26.52 & Hipp \\
\hline 5 & 79 & M & 25.66 & Hipp \\
\hline 5 & 82 & $\mathrm{~F}$ & 17.18 & Hipp \\
\hline 5 & 78 & $M$ & 15.42 & Hipp \\
\hline 5 & 102 & $\mathrm{~F}$ & 18.72 & Hipp \\
\hline 5 & 89 & M & 9.05 & Hipp \\
\hline 6 & 88 & $\mathrm{~F}$ & 15.67 & Hipp \\
\hline 6 & 89 & $\mathrm{~F}$ & 28.33 & Hipp \\
\hline 6 & 63 & $\mathrm{~F}$ & 14.12 & Hipp \\
\hline 6 & 86 & $\mathrm{~F}$ & 6 & Hipp \\
\hline
\end{tabular}

Con, control; Hipp, hippocampus; PMI, postmortem interval; M, male; $\mathrm{F}$, female.

seen in WT and $E 5^{-/-}$mice (Figure $2 \mathrm{~B}$ ). The rescued spine density in the $\mathrm{hAPP} / E 5^{-/-}$mice was largely due to an increase in spines with a mushroom-shaped, thin morphology (Figure 2C). Previous reports have shown that $E 5^{-/}$animals have increased spine density compared with that of WT animals early in development (10). By repeating these experiments, we observed similar results in mice at similar young ages (Sell et al., unpublished data). The excess spines in $E 5^{-1-}$ mice at a young age are likely pruned away to WT levels in older animals, as reported here.

Previously, $E 5^{-/-}$mice showed an increase in spine density in the CA1 of the hippocampus early in development (10). While we did not observe any change in Ephexin5 protein levels in the $\mathrm{CA} 1$ of AD mice, we conjectured that the hAPP $/ E 5^{-/-}$rescued mice would potentially show an alteration in spine density and therefore measured spine density in the CA1 stratum radiatum of our mice (Supplemental Figure 3A). While we did not observe significant variations in total spine density in the CA1 (Supplemental Figure $3 \mathrm{~B})$, there was a significant reduction in mushroom/thin subtype density in the hAPP animals that was rescued in the hAPP $/ E 5^{-/}$ animals to a degree similar to that seen in WT and $E 5^{-/-}$mice (Supplemental Figure 3C). One explanation for these findings is that $\mathrm{A} \beta$ mediates changes in the CA1 that are independent of Ephexin5 protein level changes, but can be protected from $A \beta$ by inhibiting Ephexin5 expression. Together, these results support the hypothesis that Ephexin 5 removal protects neurons against the AD-related spine deficits seen in hAPP mice.

Previous experiments have indicated that the majority of excitatory synaptic junctions are located on mushroom-shaped or thin spines (20) and therefore suggest that the rescue of these types of spines is associated with functional synapses. The observation that a reduction of Ephexin5 in $\mathrm{AD}$ mice replaces this spine type suggested to us that mice without Ephexin 5 may be protected from $\mathrm{A} \beta$-induced spine loss and maintain the mature spine morphology that is important for normal behavior. Previous studies have identified the presence in $\mathrm{AD}$ animals of learning and memory deficits (11) that are directly correlated with spine loss (21). Thus, we sought to investigate the effects of Ephexin5 removal on $\mathrm{AD}$-associated learning and memory impairment. We used novel place preference (NPP) and passive avoidance (PA), two independent behavioral paradigms known to test hippocampus-dependent spatial memory
Figure 2. Removal of Ephexin5 prevents hippocampal spine density abnormalities and cognitive deficits in hAPP mice. (A) Representative dendrite segments from WT, hAPP, hAPP/E5 $5^{-1}$, and $E 5^{-/-} \mathrm{DG}$. Scale bars: $2 \mu \mathrm{m}$. (B) DC total spine density. (C) DC mushroom/ thin spine density. (A-C) $n=3$ /genotype. (B and $\mathbf{C}$ ) ${ }^{* *} P<0.007$, by 1 -way ANOVA with Tukey's correction. (D) Object investigation in the NPP test for 2.5- to 4-month-old mice. Shown is the percentage of time spent investigating each object. The time spent with an individual object was divided by the total time spent investigating all objects and multiplied by 100 . WT $(n=12)$, hAPP $(n=11)$, hAPP/E5 $5^{--}(n=16)$, and $E 5^{-1-}$ $(n=9)$ mice were tested. (E) Latency for 6- to 8-monthold mice to enter the shock arena on days 1 and 2 . WT $(n=14)$, hAPP $(n=18)$, hAPP/E5 $5^{--}(n=12)$, and $E 5^{-/-}(n$ = 9) mice were tested. Data represent the mean \pm SEM. (D and $\mathbf{E}){ }^{* *} P<0.05$ and ${ }^{* * *} P<0.0001$. Analysis of the NPP test was performed by 1-way ANOVA with a Kruskal-Wallis correction, since the data were not normally distributed. Comparisons for the PA test were made using 2-way ANOVA with a Holm-Sidak correction.
A Representative dendrite segments

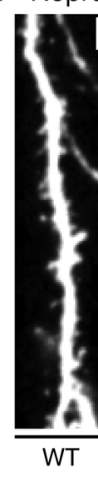

B

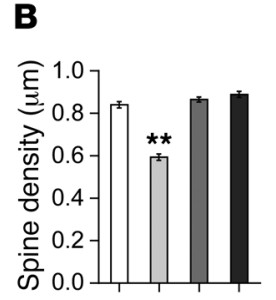

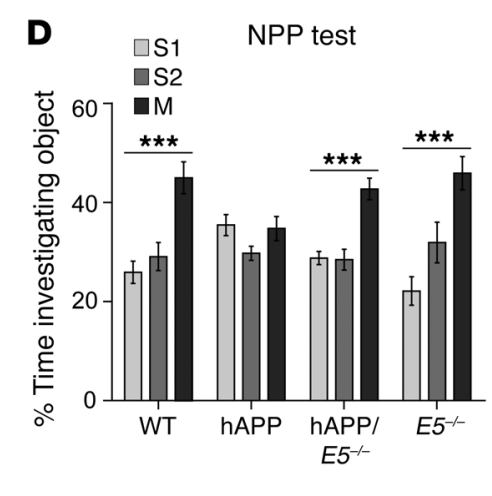

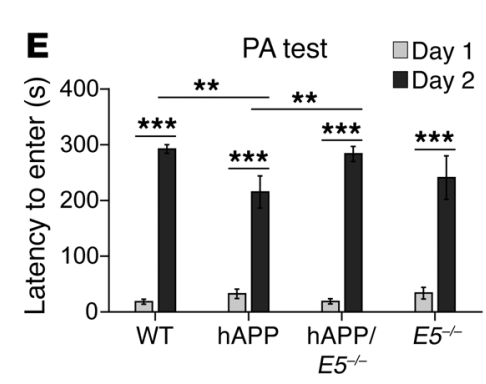

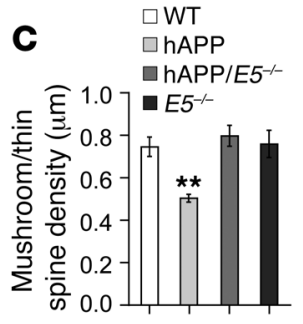


A

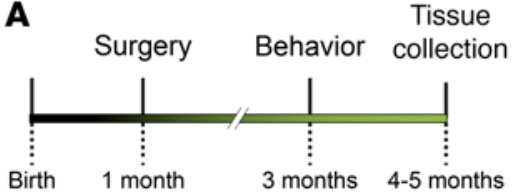

C

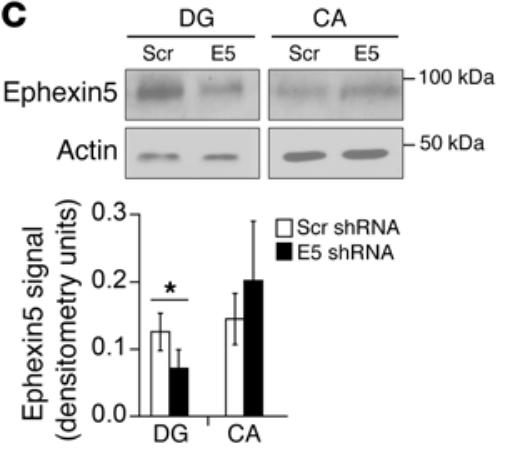

B

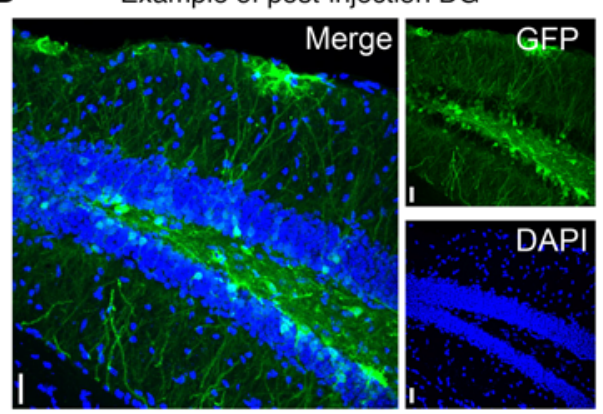

D

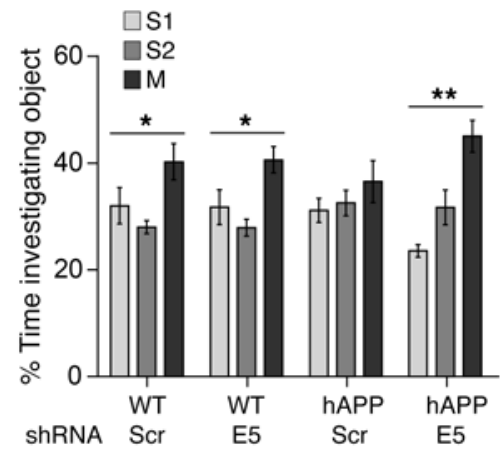

Figure 3. Lentiviral manipulation of Ephexin 5 in the DG ameliorates learning and memory deficits in developing hAPP mice. (A) Timeline for the injection of WT and AD mice with lentivirus. (B) Representative immunofluorescence staining of the DG from a mouse injected with lentivirus. Sections were stained for GFP and labeled with DAPI. Scale bars: $10 \mu \mathrm{m}$. (C) Representative Western blot and quantification of actin-normalized Ephexin5 levels in microdissected hippocampi from mice injected with lentivirus expressing shRNA against Ephexin5 (E5) or scrambled hairpin (Scr). $n=7$ mice. (D) NPP testing of object investigation for 3-month-old mice. Shown is the percentage time spent investigating each object, calculated as described in the legend for Figure 2. WT-Scr $(n=8)$, hAPP-Scr $(n=8)$, hAPP-E5 $(n=10)$, WT-E5 $(n=10)$. (C and $\mathbf{D}){ }^{*} P<0.05$ and ${ }^{* *} P<0.01$. For comparison of lentivirus-injected hippocampal Ephexin5 levels, a paired $t$ test was performed (C). Analysis of the NPP test was performed by 1-way ANOVA with a Kruskal-Wallis correction, since the data were not normally distributed (D).

(22) and associative learning (23), respectively. Published studies have shown that hAPP mice have NPP deficits (11). As expected, on the test day, the hAPP mice spent a similar amount of time investigating each object, indicating that they were unable to discriminate between the moved (M) and stationary objects (S1, S2), a task easily accomplished by the WT animals. hAPP $/ E 5^{-}$mice showed a rescued exploratory preference similar to that of WT and $E 5^{-/-}$mice, spending significantly more time with the moved object (Figure 2D), despite a lack of preference for the moved object on day 1 (data not shown). In PA testing, hAPP mice exhibited deficits (11). While mice of all genotypes showed an increased latency to enter the shock chamber 24 hours after training, hAPP mice showed a decreased latency to enter the chamber and thus impaired associative learning compared with WT and hAPP/E5 $5^{-/-}$mice (Figure 2E). The hAPP mice showed a similarly trending deficit and rescue, regardless of sex (Supplemental Figure 3, E and F). Thus, two independent behavioral tests revealed that removal of Ephexin5 in hAPP mice is sufficient to ameliorate deficits in hippocampusdependent learning and memory.

Given the amelioration of phenotypes in the hAPP $/ E 5^{-/-}$mice and previous data indicating that EPHB2 overexpression in the DG is sufficient to rescue behavioral deficits (11), we sought to deter- mine whether a reduction of Ephexin 5 in hippocampus at a presymptomatic time point was sufficient to protect against A $\beta$-induced cognitive decline. Such approaches also circumvent any possible downstream developmental compensation for $E 5$ deletion in our genetic reduction approaches, which may have confounded our results. To this end, we used lentivirus that encodes a shRNA directed against Ephexin5. This lentivirus was stereotactically injected into the DG of 4-week-old mice (Figure 3A). The investigator was double blinded to the virus and genotype of the animal. Injected mice were tested for NPP at 3 months of age. Brains were harvested from the mice at 5 months of age to determine the correct placement and spread of injection by immunofluorescence (Figure 3B) or to determine the level of Ephexin5 knockdown by Western blot analysis (Figure 3C). Mice injected with the shRNA lentivirus showed a significant decrease in DG Ephexin5 levels, with no significant change in the CA. Consistent with genetic rescue results, hAPP mice injected with the scrambled hairpin (Scr) spent the same amount of time with each object, while hAPP mice injected with the Ephexin5 hairpin (E5) showed rescued exploratory behavior similar to that of WT-Scr and WT-E5 mice, spending significantly more time with the moved object (Figure 3D). This result indicated that temporally and spatially restricted Ephexin5 reduction in a subset of granule cells in the DG of hAPP mice is sufficient to rescue the learning and memory phenotype, without affecting the WT mice in these assays. Despite only targeting subsets of neurons, these data are consistent with those of previous studies demonstrating that improving subsets of neurons can profoundly affect a larger neuronal network relevant to behavior (24).

Previous studies have suggested the importance of RhoA GEFs in the progression of $\mathrm{AD}$ and as potential drug targets to treat $\mathrm{AD}$ (25). We believe that our study is one of the few to test this hypothesis and, further, to identify a RhoA GEF, Ephexin5, as being relevant to $\mathrm{AD}$-associated dendritic spine degeneration and cognitive dysfunction. While we observed that a reduction of Ephexin 5 protected hAPP mice from developing learning and memory deficits, whether inhibition of Ephexin 5 could reverse these deficits remains to be determined. However, an intriguing feature of Ephexin5 is that its expression is very low in healthy adult tissue, is generally restricted to the hippocampus, and is found to be elevated pathologically. Molecular targets of this nature will be of great interest, as they will likely limit the off-target effects of inhibition to healthy cells. Strategies targeting late-stage AD by lowering A $\beta$ levels have had modest success in recent clinical trials (26). A bet- 
ter understanding of $A \beta$ and its effect on targets that lead to brain degeneration will likely be critical for progress in the development of $\mathrm{AD}$ therapeutics.

\section{Methods}

Detailed information can be found in the Supplemental Methods.

Statistics. Statistical analysis was performed using 2-tailed Student's $t$ tests and 1-way ANOVA with the appropriate post-hoc test. $P$ values of 0.05 or less were considered significant. All data represent the mean \pm SEM.

Study approval. All procedures were performed in accordance with an IACUC-approved protocol of The Johns Hopkins University School of Medicine.

\section{Author contributions}

GLS and SSM designed the experiments. GLS and TBS performed all experiments. GLS and SSM analyzed and interpreted the data and wrote the manuscript. TBS and SSM blinded the experiment, while GLS performed the experiments.

\section{Acknowledgments}

We thank Josh Crawford, Joseph Wardell, and Olof Lagerlof (The Johns Hopkins University School of Medicine, Baltimore, MA, USA) for reagents, technical assistance, and advice on the project. We thank the members of the Margolis laboratory for discussions and advice. We thank the Greenberg laboratory for the Ephexin5 and EPHB2 antibodies. This work was supported by institutional funding and by grants from the BrightFocus Foundation (A2012070 and R01 MH102364, to SSM). GLS was supported by a training grant from the National Eye Institute (5T32EY017203, from September 1, 2012 to August 31, 2013) and a National Science Foundation (NSF) Graduate Research Fellowship grant (no. 1232825).

Address correspondence to: Seth S. Margolis, The Johns Hopkins School of Medicine, Department of Biological Chemistry, Wood Basic Science Building Room 517, 725 N. Wolfe Street, Baltimore, Maryland 21205, USA. Phone: 410.502.5362; E-mail: smargol7@ jhmi.edu.
1. Kawas CH. Clinical practice. Early Alzheimer's disease. NEngl JMed. 2003;349(11):1056-1063.

2. Selkoe DJ. Alzheimer's disease: genes, proteins, and therapy. Physiol Rev. 2001;81(2):741-766.

3. Gandy S. The role of cerebral amyloid beta accumulation in common forms of Alzheimer disease. J Clin Invest. 2005;115(5):1121-1129.

4. Morishima Y, et al. Beta-amyloid induces neuronal apoptosis via a mechanism that involves the c-Jun $\mathrm{N}$-terminal kinase pathway and the induction of Fas ligand. J Neurosci. 2001;21(19):7551-7560.

5. Coleman PD, Yao PJ. Synaptic slaughter in Alzheimer's disease. Neurobiol Aging. 2003;24(8):1023-1027.

6. Terry RD, et al. Physical basis of cognitive alterations in Alzheimer's disease: synapse loss is the major correlate of cognitive impairment. Ann Neurol. 1991;30(4):572-580.

7. Lue LF, et al. Soluble amyloid beta peptide concentration as a predictor of synaptic change in Alzheimer's disease. Am J Pathol. 1999;155(3):853-862.

8. Lacor PN, et al. Synaptic targeting by Alzheimer'srelated amyloid beta oligomers. J Neurosci. 2004;24(45):10191-10200.

9. Blalock EM, Geddes JW, Chen KC, Porter NM, Markesbery WR, Landfield PW. Incipient Alzheimer's disease: microarray correlation analyses reveal major transcriptional and tumor suppressor responses. Proc Natl Acad Sci USA. 2004;101(7):2173-2178.
10. Margolis SS, et al. EphB-mediated degradation of the RhoA GEF Ephexin5 relieves a developmental brake on excitatory synapse formation. Cell. 2010;143(3):442-455.

11. Cissé M, et al. Reversing EphB2 depletion rescues cognitive functions in Alzheimer model. Nature. 2011;469(7328):47-52.

12. Simón AM, et al. Early changes in hippocampal Eph receptors precede the onset of memory decline in mouse models of Alzheimer's disease. JAlzheimers Dis. 2009;17(4):773-786.

13. Kayser MS, Nolt MJ, Dalva MB. EphB receptors couple dendritic filopodia motility to synapse formation. Neuron. 2008;59(1):56-69.

14. Llorens-Martín M, et al. Selective alterations of neurons and circuits related to early memory loss in Alzheimer's disease. Front Neuroanat. 2014;8:38.

15. Smith DL, Pozueta J, Gong B, Arancio O, Shelanski M. Reversal of long-term dendritic spine alterations in Alzheimer disease models. Proc Natl Acad Sci U S A. 2009;106(39):16877-16882.

16. Moolman DL, Vitolo OV, Vonsattel JP, Shelanski ML. Dendrite and dendritic spine alterations in Alzheimer models. J Neurocytol. 2004;33(3):377-387.

17. Pozueta J, Lefort R, Ribe EM, Troy CM, Arancio O, Shelanski M. Caspase-2 is required for dendritic spine and behavioural alterations in $\mathrm{J} 20$ APP transgenic mice. Nat Commun. 2013;4:1939.

18. Harris JA, et al. Many neuronal and behavioral impairments in transgenic mouse models of
Alzheimer's disease are independent of caspase cleavage of the amyloid precursor protein. $\mathrm{J} \mathrm{Neu}$ rosci. 2010;30(1):372-381.

19. Jacobsen JS, et al. Early-onset behavioral and synaptic deficits in a mouse model of Alzheimer's disease. Proc Natl Acad Sci US A. 2006;103(13):5161-5166.

20. Fiala JC, Feinberg M, Popov V, Harris KM. Synaptogenesis via dendritic filopodia in developing hippocampal area CA1. J Neurosci. 1998;18(21):8900-8911.

21. Chen Y, et al. Correlated memory defects and hippocampal dendritic spine loss after acute stress involve corticotropin-releasing hormone signaling. Proc Natl Acad Sci U S A. 2010;107(29):13123-13128.

22. Barker GR, Warburton EC. When is the hippocampus involved in recognition memory? J Neurosci. 2011;31(29):10721-10731.

23. van der Poel AM. Ethological study of the behaviour of the albino rat in a passive-avoidance test. Acta Physiol Pharmacol Neerl. 1967;14(4):503-505.

24. Li CY, Poo MM, Dan Y. Burst spiking of a single cortical neuron modifies global brain state. Science. 2009;324(5927):643-646.

25. Lefort R. Reversing synapse loss in Alzheimer's disease: Rho-guanosine triphosphatases and insights from other brain disorders. Neurotherapeutics. 2015;12(1):19-28.

26. Huang Y, Mucke L. Alzheimer mechanisms and therapeutic strategies. Cell. 2012;148(6):1204-1222. 